\title{
ESTIMATION OF PARAMETERS OF A MIXTURE OF TWO SYMMETRIC DISTRIBUTIONS FROM A BIASED SAMPLE
}

UDC 519.21

\author{
T. GORBACH
}

\begin{abstract}
We consider a biased sample from a mixture of two symmetric distributions that differ by a shift parameter. The method of moments and the generalized estimating equations method are used to estimate unknown parameters. Adaptive estimators are constructed by using the estimators of optimal estimating functions and those obtained by the method of moments. The asymptotic behavior of GEEestimators and adaptive estimators is investigated.
\end{abstract}

\section{INTRODUCTION}

We consider the semiparametric estimation of parameters of a mixture of two symmetric distributions constructed from a biased sample. Many papers are devoted to the estimation of parameters of a model of mixtures; see, for example, [1] and [2. The case of a mixture of two symmetric distributions and an unbiased sample is considered in [3]- 6 . The method of moments estimators for parameters of a mixture constructed from an unbiased sample are studied in [3]. The conditions for the consistency and asymptotic normality of such estimators are also obtained in [3. The asymptotic variance of these estimators is found in 3 .

The estimators are constructed in [4 with the help of the generalized estimating equations method (referred to as GEE-estimators in what follows) for parameters of a mixture of two symmetric distributions and for an unbiased sample. Asymptotic normality is proved and the lower bound for their dispersion coefficients is found in 44. Adaptive estimators of unknown parameters of a mixture of two distributions are constructed in [5] in the case of an unbiased sample. The asymptotic behavior of such estimators is also investigated in [5]. The results obtained in [3] and [5] are generalized in the current paper to the case of a biased sample.

The estimation of distribution functions from a biased homogeneous sample is described in [7, p. 286]. We follow a different approach based on the Horwitz-Thompson estimator (see [8, p. 196] or [9, p. 31]) and on the technique of the asymptotic analysis of GEE-estimators described in [7].

The notion of a biased sample and setting of the problem are described in Section 2 , The method of moments estimators and GEE-estimators of parameters of a mixture constructed from a biased sample are considered in Sections 3 and 4, respectively, for the estimating functions $\sum \beta_{i} u_{i}$, where the $u_{i}$ are fixed basis functions. Conditions for the asymptotic normality of GEE-estimators are studied in Section 5. The optimal coefficients $\beta_{i}^{*}$ for which $\Sigma\left(\beta_{i}^{*}\right)$ corresponding to the asymptotic covariance matrix of

2010 Mathematics Subject Classification. Primary 62G05; Secondary 62G20.

Key words and phrases. Biased sample, mixture of two symmetric distributions, generalized estimating equations, adaptive estimators. 
GEE-estimators attains its minimal value are also found in Section 5. Since the optimal coefficients $\beta_{i}^{*}$ depend on unknown parameters of a sample, we construct consistent estimators $\hat{\beta}_{i}$ for $\beta_{i}^{*}$ in Section 6 .

In Section 7, we construct adaptive estimators with the asymptotic covariance matrix $\Sigma\left(\beta_{i}^{*}\right)$ with the help of moments estimators and of estimators $\hat{\beta}_{i}$ corresponding to the optimal coefficients of estimating functions.

\section{Setting of the PRoblem}

Consider a sample $X=\left\{\xi_{1}, \ldots, \xi_{n}\right\}$ of some elements of a population. We are interested in the distribution of some characteristic $\eta(O)$ of elements of this population. The sample is biased; that is, the probability of an element to be sampled depends on the characteristic $\eta(O)$. We consider the case where

$$
\mathrm{P}(\text { element } O \text { is sampled } \mid \eta(O)=t)=w(t),
$$

$w(t) \neq 0$ for all $t$ of the support of the distribution $F$, where $F$ is the distribution function of elements $\eta(O)$ of the population. In what follows we assume that $F$ is a mixture of two distributions that differ by a shift parameter and that $F$ possesses the density

$$
\eta \sim p f\left(x-a_{1}\right)+(1-p) f\left(x-a_{2}\right),
$$

where $0<p<\frac{1}{2}$ is the concentration of the first component in the mixture, $a_{i} \in \mathbf{R}$, $i=1,2$ and where $a_{1} \neq a_{2}$ are medians of the components of the mixture, and $f$ is a symmetric density of the deviation between observations and the median $(f(-x)=f(x))$. Here $f$ is an unknown differentiable function.

According to Example 5.2 in [7, the distribution function of the random variables $\xi_{i}$, $i=1, \ldots, n$, equals

$$
G(t)=\mathrm{P}\{\eta(O)<t \mid O \text { is sampled }\}=\frac{\int_{-\infty}^{t} w(x) F(d x)}{\int_{-\infty}^{\infty} w(x) F(d x)} .
$$

Thus $\left(\xi_{1}, \ldots, \xi_{n}\right)$ is a sample of independent identically distributed random variables with the distribution function $G$ constructed from $F$ as in (2) (here $F$ is defined by (1)).

It is usually the case that the parameters $p, a_{1}$, and $a_{2}$ are unknown. The problem is to estimate the unknown parameter $\theta=\left(p, a_{1}, a_{2}\right)$ from the sample $X=\left\{\xi_{1}, \ldots, \xi_{n}\right\}$.

As in the case of the Horwitz-Thompson estimator (see [9, Section 1.7]), one can use

$$
\hat{h}_{n}(\eta)=\frac{1}{\frac{1}{n} \sum_{j=1}^{n} \frac{1}{w\left(\xi_{j}\right)}} \frac{1}{n} \sum_{j=1}^{n} \frac{1}{w\left(\xi_{j}\right)} h\left(\xi_{j}\right)
$$

to estimate the expectation of an arbitrary function $h(\eta)$ from a biased sample $X=$ $\left\{\xi_{1}, \ldots, \xi_{n}\right\}$ if the expectation $\mathrm{E} h(\eta)$ exists.

\section{Method of moments estimators}

FOR PARAMETERS CONSTRUCTED FROM A BIASED SAMPLE

The construction of the method of moments estimators for parameters of a mixture from an unbiased sample is described in 3 . The procedure remains essentially the same in the case of a biased sample. The Horwitz-Thompson estimator $\hat{y}_{k}$ constructed from a biased sample is used to estimate theoretical moments,

$$
\hat{y}_{k}=\frac{1}{\frac{1}{n} \sum_{i=1}^{n} \frac{1}{w\left(\xi_{i}\right)}} \frac{1}{n} \sum_{i=1}^{n} \frac{1}{w\left(\xi_{i}\right)} \xi_{i}^{k} .
$$

If $\mathrm{E}\left(\left|\eta_{1}\right|^{k}\right)<\infty$, then $\hat{y}_{k}$ is a consistent estimator for $\mathrm{E} \eta^{k}$. 
The method of moments estimators for parameters $p, a_{1}$, and $a_{2}$ are

$$
\hat{p}_{n}=\hat{p}\left(C_{n}\right)= \begin{cases}P_{1}\left(C_{n}\right), & C_{n}>432 \\ \frac{1}{2}-\frac{\sqrt{2}}{3}, & C_{n}=432 \\ P_{2}\left(C_{n}\right), & C_{n}<432,\end{cases}
$$

$$
\begin{gathered}
\hat{a}_{1, n}=\hat{y}_{1}-\frac{\sqrt[3]{\left(1-3 \hat{p}_{n}+2 \hat{p}_{n}^{2}\right)^{2}\left(3 \hat{y}_{1} \hat{y}_{2}-2 \hat{y}_{1}^{3}-\hat{y}_{3}\right)}}{\sqrt[3]{\hat{p}_{n}}\left(1-2 \hat{p}_{n}\right)} \\
\hat{a}_{2, n}=\frac{1}{1-\hat{p}_{n}}\left(\hat{y}_{1}-\hat{p}_{n} \hat{a}_{1, n}\left(\hat{p}_{n}\right)\right)
\end{gathered}
$$

where

$$
\begin{gathered}
C_{n}=\frac{\left(24 \hat{y}_{1}^{5}-60 \hat{y}_{1}^{3} \hat{y}_{2}+30 \hat{y}_{1} \hat{y}_{2}^{2}+20 \hat{y}_{1}^{2} \hat{y}_{3}-10 \hat{y}_{2} \hat{y}_{3}-5 \hat{y}_{1} \hat{y}_{4}+\hat{y}_{5}\right)^{3}}{\left(2 \hat{y}_{1}^{3}-3 \hat{y}_{1} \hat{y}_{2}+\hat{y}_{3}\right)^{5}}, \\
P_{1}(C)=\frac{1}{2}-\frac{\sqrt{6}}{12} \sqrt{4+\frac{2 C(-\cos (B(C))+\sqrt{3} \sin (B(C)))}{\sqrt{(-432+C) C}},} \\
P_{2}(C)=\frac{1}{2}-\frac{\sqrt{-(C+A(C))^{2}+432(C+2 A(C))}}{2 \sqrt{3} \sqrt{(432-C) A(C)}}, \\
A(C)=\sqrt[3]{-(-432+C)^{2} C+12 \sqrt{3} \sqrt{(432-C)^{3} C^{2}}}, \\
B(C)=1 / 3\left(\pi-\arctan \left(\frac{12 \sqrt{3}}{\sqrt{-432+C}}\right)\right) .
\end{gathered}
$$

Theorem 3.1. If

1. $\mathrm{E}\left|\eta_{1}\right|^{5}<\infty$,

2. $a_{1} \neq a_{2}$,

3. $p \in(0,1 / 2)$,

then estimators (4) -(6) are strictly consistent.

The proof of Theorem 3.1 is similar to that of Theorem 3.2 of $[3]$.

Remark 3.1. Restrictions 2 and 3 of Theorem 3.1 are, in fact, the identifiability conditions. If $p=1 / 2$ or $a_{1}=a_{2}$, then the estimation of parameters is, generally speaking, a nonidentifiable problem. The case $p \in\left(\frac{1}{2} ; 1\right)$ is also treated in Theorem 3.1 since it turns to the case of $p \in(0,1 / 2)$ by reverting the components. Restriction 3 tells us that the second component has a higher concentration in the mixture.

\section{GEE-estimators For PARAmeters}

OF A MIXTURE CONSTRUCTED FROM A BIASED SAMPLE

We apply the method of generalized estimating equations to estimate parameters $p$, $a_{1}$, and $a_{2}$. This method for unbiased samples is described in [5].

The construction of the GEE-estimators starts with choosing a triple of arbitrary odd functions $g=g_{i}(x), i=1,2,3$, such that $\mathrm{E} g_{i}(\eta-\alpha)<\infty$ for all $\alpha \in \mathbf{R}$. Then

$$
\mathrm{E} g_{i}(\eta-\alpha)=p \int_{-\infty}^{\infty} g_{i}(x) f\left(x+\alpha-a_{1}\right) d x+(1-p) \int_{-\infty}^{\infty} g_{i}(x) f\left(x+\alpha-a_{2}\right) d x
$$

and

$$
\mathrm{E}\left[p g\left(\eta-a_{1}\right)+(1-p) g\left(\eta-a_{2}\right)\right]=0
$$


Throughout this paper we consider the estimating functions

$$
g_{i}(x)=\sum_{m=1}^{M} \beta_{i m} u_{m}(x)
$$

where $\left\{u_{m}(x)\right\}_{m=1}^{M}$ is a certain set of $M$ odd basis functions.

A statistic $\hat{\theta}_{n}=\left(\hat{p}_{n}, \hat{a}_{1, n}, \hat{a}_{2, n}\right)$ is called a GEE-estimator of a parameter $\theta=\left(p, a_{1}, a_{2}\right)$ with an estimating triplet $\left(g_{1}, g_{2}, g_{3}\right)$ if the equations

$$
\pi \hat{g}_{i}\left(\alpha_{1}\right)+(1-\pi) \hat{g}_{i}\left(\alpha_{2}\right)=0, \quad i=1,2,3,
$$

hold almost surely with $\pi=\hat{p}_{n}, \alpha_{1}=\hat{a}_{1, n}$, and $\alpha_{2}=\hat{a}_{2, n}$.

Here $\hat{g}_{i}$ is the Horwitz-Thompson estimator for $g_{i}$ constructed from a biased sample:

$$
\hat{g}(\alpha)=\frac{1}{\frac{1}{n} \sum_{j=1}^{n} \frac{1}{w\left(\xi_{j}\right)}} \frac{1}{n} \sum_{j=1}^{n} \frac{1}{w\left(\xi_{j}\right)} g\left(\xi_{j}-\alpha\right) .
$$

Substituting estimators of $g$ into (8) we obtain

$$
\frac{1}{\frac{1}{n} \sum_{j=1}^{n} \frac{1}{w\left(\xi_{j}\right)}} \frac{1}{n} \sum_{j=1}^{n} \frac{\pi g_{i}\left(\xi_{j}-\alpha_{1}\right)+(1-\pi) g_{i}\left(\xi_{j}-\alpha_{2}\right)}{w\left(\xi_{j}\right)}=0, \quad i=1, \ldots, 3 .
$$

Simplifying the latter expression we get

$$
\frac{1}{n} \sum_{j=1}^{n} \frac{\pi g_{i}\left(\xi_{j}-\alpha_{1}\right)+(1-\pi) g_{i}\left(\xi_{j}-\alpha_{2}\right)}{w\left(\xi_{j}\right)}=0, \quad i=1, \ldots, 3 .
$$

This is the GEE-equation in the case of biased samples.

Put

$$
\begin{gathered}
\nu=\left(\pi, \alpha_{1}, \alpha_{2}\right), \\
\mathbf{g}=\left(g_{1}, g_{2}, g_{3}\right)^{T}, \\
\mathbf{h}\left(\xi_{j}, \nu\right)=\frac{\pi \mathbf{g}\left(\xi_{j}-\alpha_{1}\right)+(1-\pi) \mathbf{g}\left(\xi_{j}-\alpha_{2}\right)}{w\left(\xi_{j}\right)} .
\end{gathered}
$$

If the true value $\theta=\left(p, a_{1}, a_{2}\right)$ is a root of the equation

$$
\mathrm{E} \mathbf{g}\left(\xi_{j}, \nu\right)=0
$$

then the corresponding GEE-estimator $\hat{\theta}_{n}$ of parameters of a mixture is consistent. The method of moments estimator is an example of GEE-estimators in the case of a mixture.

\section{Asymptotic normality of GEE-EStimators}

The asymptotic normality of GEE-estimators for an unbiased estimator of jointly independent random variables is considered in [7. The asymptotic normality of GEEestimators for parameters of a mixture of two distributions for an unbiased sample is studied in [4] and [5]. We prove the asymptotic normality of GEE-estimators for parameters of a mixture of two distributions in the case of a biased sample.

Put $V(\theta)=-\mathbf{E} \frac{\partial \mathbf{h}\left(\xi_{j}, \theta\right)}{\partial \theta}, Q(\theta)=\operatorname{Cov}\left(\mathbf{h}\left(\xi_{j}, \theta\right)\right)$.

Theorem 5.1. Assume that

1. the derivatives $f^{\prime}(x)$ and $u_{m}^{\prime}(x), m=1, \ldots, M$, are continuous;

2. $\hat{\theta}_{n}=\left(\hat{p}_{n}, \hat{a}_{1, n}, \hat{a}_{2, n}\right)^{T}$ is a consistent estimator;

3. $\mathrm{E} \frac{u_{m}^{2}\left(\xi_{1}-a_{k}\right)}{w^{2}\left(\xi_{1}\right)}<\infty, m=1, \ldots, M$ and $k=1,2$;

4. the elements of the matrix $V$ are finite; $\operatorname{det} V \neq 0$; 
5.

$$
\begin{gathered}
\exists \varepsilon>0, \delta>0: \quad \mathrm{E} \sup _{\alpha:\left|\alpha-a_{k}\right|<\varepsilon}\left|\frac{u_{m}\left(\xi_{1}-\alpha\right)}{w\left(\xi_{1}\right)}\right|^{1+\delta}<\infty ; \\
\mathrm{E} \sup _{\alpha:\left|\alpha-a_{k}\right|<\varepsilon}\left|\frac{u_{m}^{\prime}\left(\xi_{1}-\alpha\right)}{w\left(\xi_{1}\right)}\right|^{1+\delta}<\infty, \quad m=1, \ldots, M \text { and } k=1,2 ;
\end{gathered}
$$

6. $u_{m}(x+\delta) f(x) \rightarrow 0$ as $x \rightarrow \pm \infty$ for all $\delta \in \mathbf{R}$;

7. $w(x)>0, x \in \operatorname{supp}(F)$.

Then

$$
\sqrt{n}\left(\hat{\theta}_{n}-\theta\right) \stackrel{d}{\rightarrow} N(0, \Sigma),
$$

where $\Sigma=\Sigma\left(g_{1}, g_{2}, g_{3}\right)=V(\theta)^{-1} Q(\theta)\left(V(\theta)^{-1}\right)^{T}$.

Theorem 5.1 is a corollary of Theorem 5.14 in [7].

The matrices $V$ and $Q$ are evaluated explicitly. Let

$$
I=\int_{-\infty}^{\infty} w(x) f(x) d x
$$

Since

$$
\frac{\partial \mathrm{E}_{t} \mathbf{h}\left(\xi_{1}, t\right)}{\partial t}=0
$$

we have

$$
\begin{aligned}
\int_{-\infty}^{\infty} & p \frac{g^{\prime}\left(x-a_{1}\right)}{w(x)} w(x)\left[p f\left(x-a_{1}\right)+(1-p) f\left(x-a_{2}\right)\right] d x \\
& =-\int_{-\infty}^{\infty}\left[\frac{p g\left(x-a_{1}\right)+(1-p) g\left(x-a_{2}\right)}{w(x)}\right] w(x) p f^{\prime}\left(x-a_{1}\right) d x
\end{aligned}
$$

Similarly,

$$
\begin{gathered}
\int_{-\infty}^{\infty}(1-p) \frac{g^{\prime}\left(x-a_{2}\right)}{w(x)} w(x)\left[p f\left(x-a_{1}\right)+(1-p) f\left(x-a_{2}\right)\right] d x \\
=-\int_{-\infty}^{\infty}\left[p g\left(x-a_{1}\right)+(1-p) g\left(x-a_{2}\right)\right](1-p) f^{\prime}\left(x-a_{2}\right) d x \\
\int_{-\infty}^{\infty} \frac{g\left(x-a_{1}\right)-g\left(x-a_{2}\right)}{w(x)} w(x)\left[p f\left(x-a_{1}\right)+(1-p) f\left(x-a_{2}\right)\right] d x \\
=-\int_{-\infty}^{\infty} \frac{p g\left(x-a_{1}\right)-(1-p) g\left(x-a_{2}\right)}{w(x)} w(x)\left[f\left(x-a_{1}\right)-f\left(x-a_{2}\right)\right] d x
\end{gathered}
$$

Using the above relations, we conclude that

$$
V=-\frac{1}{I}\left(\begin{array}{lll}
\int_{-\infty}^{\infty} g_{1} b_{1} & \int_{-\infty}^{\infty} g_{1} b_{2} & \int_{-\infty}^{\infty} g_{1} b_{3} \\
\int_{-\infty}^{\infty} g_{2} b_{1} & \int_{-\infty}^{\infty} g_{2} b_{2} & \int_{-\infty}^{\infty} g_{2} b_{3} \\
\int_{-\infty}^{\infty} g_{3} b_{1} & \int_{-\infty}^{\infty} g_{3} b_{2} & \int_{-\infty}^{\infty} g_{3} b_{3}
\end{array}\right)
$$

where

$$
\begin{gathered}
b_{1}(x)=p^{2} f^{\prime}(x)+p(1-p) f^{\prime}\left(x+a_{2}-a_{1}\right), \\
b_{2}(x)=p(1-p) f^{\prime}\left(x+a_{1}-a_{2}\right)+(1-p)^{2} f^{\prime}(x), \\
b_{3}(x)=(2 p-1) f(x)-p f\left(x+a_{1}-a_{2}\right)+(1-p) f\left(x+a_{2}-a_{1}\right) .
\end{gathered}
$$




$$
\begin{aligned}
Q= & \operatorname{Cov}\left(\mathbf{h}\left(\xi_{j}, \nu\right)\right) \\
=( & \mathrm{E}\left(\frac{p g_{i}\left(\xi_{1}-a_{1}\right)+(1-p) g_{i}\left(\xi_{1}-a_{2}\right)}{w\left(\xi_{1}\right)}\right) \\
& \left.\times\left(\frac{p g_{j}\left(\xi_{1}-a_{1}\right)+(1-p) g_{j}\left(\xi_{1}-a_{2}\right)}{w\left(\xi_{1}\right)}\right)\right)_{i, j=1}^{3}
\end{aligned}
$$

We are going to find the functions $g_{1}, g_{2}$, and $g_{3}$ for which the minimal value of the covariance matrix $\Sigma$ is attained.

We rewrite the matrices $V$ and $Q$ with the help of the expansion in the basis (7). Let

$$
\begin{aligned}
& b_{k}^{+}=-\frac{1}{I} \int_{-\infty}^{\infty} b_{1}(x) u_{k}(x) d x, \\
& b_{k}^{-}=-\frac{1}{I} \int_{-\infty}^{\infty} b_{2}(x) u_{k}(x) d x, \\
& d_{k}=-\frac{1}{I} \int_{-\infty}^{\infty} b_{3}(x) u_{k}(x) d x .
\end{aligned}
$$

Then

$$
\begin{gathered}
V=\left(\begin{array}{ccc}
\sum_{k=1}^{M} \beta_{1 k} b_{k}^{+} & \sum_{k=1}^{M} \beta_{1 k} b_{k}^{-} & \sum_{k=1}^{M} \beta_{1 k} d_{k} \\
\sum_{k=1}^{M} \beta_{2 k} b_{k}^{+} & \sum_{k=1}^{M} \beta_{2 k} b_{k}^{-} & \sum_{k=1}^{M} \beta_{2 k} d_{k} \\
\sum_{k=1}^{M} \beta_{3 k} b_{k}^{+} & \sum_{k=1}^{M} \beta_{3 k} b_{k}^{-} & \sum_{k=1}^{M} \beta_{3 k} d_{k}
\end{array}\right) \\
Q=\left(\sum_{k, l=1}^{M} c_{k l} \beta_{i k} \beta_{j l}\right)_{i, j=1}^{3}
\end{gathered}
$$

where

$$
\begin{aligned}
c_{k l}=(\mathrm{E} & \left(\frac{p u_{k}\left(\xi_{1}-a_{1}\right)+(1-p) u_{k}\left(\xi_{1}-a_{2}\right)}{w\left(\xi_{1}\right)}\right) \\
& \left.\times\left(\frac{p u_{l}\left(\xi_{1}-a_{1}\right)+(1-p) u_{l}\left(\xi_{1}-a_{2}\right)}{w\left(\xi_{1}\right)}\right)\right)_{i, j=1}^{3} .
\end{aligned}
$$

Put

$$
C=\left(c_{k l}\right)_{k, l=1}^{M}, \quad B=\left(\begin{array}{ccc}
b_{1}^{+} & b_{1}^{-} & d_{1} \\
\vdots & \vdots & \vdots \\
b_{M}^{+} & b_{M}^{-} & d_{M}
\end{array}\right), \quad \beta=\left(\begin{array}{ccc}
\beta_{11} & \beta_{21} & \beta_{31} \\
\vdots & \vdots & \vdots \\
\beta_{1 M} & \beta_{2 M} & \beta_{3 M}
\end{array}\right)
$$

Theorem 3.2 of 5 implies that the minimal value of the limit covariance matrix $\Sigma$ is attained at $\beta^{*}=\left(B^{T} C^{-1} B\right)^{-1} C^{-1} B$ and equals $\Sigma\left(\beta^{*}\right)=\left(B^{T} C^{-1} B\right)^{-1}$. In other words,

$$
\Sigma(\beta) \geq \Sigma\left(\beta^{*}\right)
$$

for all $\beta$.

Remark 5.1. The inequality $\Sigma(\beta) \geq \Sigma\left(\beta^{*}\right)$ means that the matrix $\Sigma(\beta)-\Sigma\left(\beta^{*}\right)$ is nonnegative definite. 


\section{Estimators For the OPtimal COEFFicients $\beta^{*}$}

The matrices $B$ and $C$ as well as the asymptotic covariance matrix $\Sigma$ depend on unknown parameters $p, a_{1}$, and $a_{2}$. Thus we need to find consistent estimators for parameters of the matrices $B$ and $C$.

By construction of the matrix $V$,

The estimators for these parameters are

$$
\begin{gathered}
b_{k}^{+}=p \mathrm{E} \frac{u_{k}^{\prime}\left(\xi_{1}-a_{1}\right)}{w\left(\xi_{1}\right)}, \quad b_{k}^{-}=(1-p) \mathrm{E} \frac{u_{k}^{\prime}\left(\xi_{1}-a_{2}\right)}{w\left(\xi_{1}\right)}, \\
d_{k}=\mathrm{E}\left[\frac{u_{k}\left(\xi_{1}-a_{2}\right)-u_{k}\left(\xi_{1}-a_{1}\right)}{w\left(\xi_{1}\right)}\right] .
\end{gathered}
$$

$$
\begin{gathered}
\hat{b}_{k, n}^{+}=\hat{p}_{n} \frac{1}{n} \sum_{j=1}^{n} \frac{u_{k}^{\prime}\left(\xi_{j}-\hat{a}_{1, n}\right)}{w\left(\xi_{j}\right)}, \\
\hat{b}_{k, n}^{-}=\hat{p}_{n} \frac{1}{n} \sum_{j=1}^{n} \frac{u_{k}^{\prime}\left(\xi_{j}-\hat{a}_{2, n}\right)}{w\left(\xi_{j}\right)}, \\
\hat{d}_{k, n}=\frac{1}{n} \sum_{j=1}^{n} \frac{u_{k}\left(\xi_{j}-\hat{a}_{2, n}\right)-u_{k}\left(\xi_{j}-\hat{a}_{1, n}\right)}{w\left(\xi_{j}\right)}, \\
\hat{c}_{k l, n}=\frac{1}{n} \sum_{j=1}^{n} \frac{\hat{p}_{n} u_{k}\left(\xi_{j}-\hat{a}_{1, n}\right)+\left(1-\hat{p}_{n}\right) u_{k}\left(\xi_{j}-\hat{a}_{2, n}\right)}{w\left(\xi_{j}\right)} \\
\times \frac{\hat{p}_{n} u_{l}\left(\xi_{j}-\hat{a}_{1, n}\right)+\left(1-\hat{p}_{n}\right) u_{l}\left(\xi_{j}-\hat{a}_{2, n}\right)}{w\left(\xi_{j}\right)},
\end{gathered}
$$

where $\hat{p}_{n}, \hat{a}_{1, n}$, and $\hat{a}_{2, n}$ are moment estimators of the parameters of the mixture. Recall that moment estimators are consistent.

Theorem 6.1. Assume that $\Theta_{1}$ and $\Theta_{2}$ in a neighborhood of zero are such that

1. $\mathrm{E}\left(\sup _{\gamma \in \Theta_{1}} \frac{u_{k}^{\prime}\left(\xi_{1}-a_{i}+\gamma\right)}{w\left(\xi_{1}\right)}\right)^{2}<\infty, i=1,2, k=1, M$;

2. $\operatorname{Esup}_{\gamma \in \Theta_{2}}\left|\frac{u_{k}^{\prime \prime}\left(\xi_{1}-a_{i}+\gamma\right)}{w\left(\xi_{1}\right)}\right|<\infty, i=1,2, k=1, M$;

3. $\mathrm{E}\left(\sup _{\gamma \in \Theta_{1}} \frac{u_{k}\left(\xi_{1}-a_{i}+\gamma\right)}{w\left(\xi_{1}\right)}\right)^{2}<\infty, i=1,2, k=1, M$;

4. $w(x)>0, x \in \operatorname{supp}(F)$.

Then the estimators $\hat{b}_{k, n}^{+}, \hat{b}_{k, n}^{-}, \hat{d}_{k, n}$, and $\hat{c}_{k l, n}$ are consistent.

Proof. Consider

$$
\hat{b}_{k, n}^{+}-b_{k}^{+}=\hat{p}_{n} \frac{1}{n} \sum_{j=1}^{n} \frac{u_{k}^{\prime}\left(\xi_{j}-\hat{a}_{1, n}\right)}{w\left(\xi_{j}\right)}-p \mathrm{E} \frac{u_{k}^{\prime}\left(\xi_{1}-a_{1}\right)}{w\left(\xi_{1}\right)}=\varepsilon_{1, n}+\varepsilon_{2, n}+\varepsilon_{3, n},
$$

where

$$
\begin{gathered}
\varepsilon_{1, n}=\left(\hat{p}_{n}-p\right) \frac{1}{n} \sum_{j=1}^{n} \frac{u_{k}^{\prime}\left(\xi_{j}-\hat{a}_{1, n}\right)}{w\left(\xi_{j}\right)}=\left(\hat{p}_{n}-p\right) \frac{1}{n} \sum_{j=1}^{n} \frac{u_{k}^{\prime}\left(\xi_{j}-a_{1}+a_{1}-\hat{a}_{1, n}\right)}{w\left(\xi_{j}\right)}, \\
\varepsilon_{2, n}=p \frac{1}{n} \sum_{j=1}^{n} \frac{u_{k}^{\prime}\left(\xi_{j}-\hat{a}_{1, n}\right)-u_{k}^{\prime}\left(\xi_{j}-a_{1}\right)}{w\left(\xi_{j}\right)} \\
\varepsilon_{3, n}=p \frac{1}{n} \sum_{j=1}^{n} \frac{u_{k}^{\prime}\left(\xi_{j}-a_{1}\right)}{w\left(\xi_{j}\right)}-p \mathrm{E} \frac{u_{k}^{\prime}\left(\xi_{1}-a_{1}\right)}{w\left(\xi_{1}\right)}
\end{gathered}
$$


Since the moment estimator $\hat{p}_{n}$ is consistent, $\hat{p}_{n}-p \rightarrow 0$ in probability. We are going to show that the sequence

$$
S_{n}=\frac{1}{n} \sum_{j=1}^{n} \frac{u_{k}^{\prime}\left(\xi_{j}-a_{1}+a_{1}-\hat{a}_{1, n}\right)}{w\left(\xi_{j}\right)}
$$

is stochastically bounded. In other words, we show that $\sup _{n} \mathrm{P}\left(\left|S_{n}\right|>C\right) \rightarrow 0$ as $C \rightarrow \infty$. Indeed,

$$
\mathrm{P}\left(\left|S_{n}\right|>C\right)=\mathrm{P}\left(\left|S_{n}\right|>C,\left(a_{1}-\hat{a}_{1, n}\right) \notin \Theta_{1}\right)+\mathrm{P}\left(\left|S_{n}\right|>C,\left(a_{1}-\hat{a}_{1, n}\right) \in \Theta_{1}\right) .
$$

The first term approaches zero as $n \rightarrow \infty$ since $\hat{a}_{1, n}$ is a consistent estimator of $a_{1}$.

Consider the second term. By Chebyshev's inequality and restriction 1,

$$
\begin{aligned}
& \mathrm{P}\left(\left|S_{n}\right|>C,\left(a_{1}-\hat{a}_{1, n}\right) \in \Theta_{1}\right)<\mathrm{P}\left(\frac{1}{n} \sum_{j=1}^{n} \sup _{\gamma \in \Theta_{1}}\left|\frac{u_{k}^{\prime}\left(\xi_{j}-a_{i}+\gamma\right)}{w\left(\xi_{j}\right)}\right|>C\right) \\
& <\frac{\operatorname{Esup}_{\gamma \in \Theta_{1}}\left|\frac{u_{k}^{\prime 2}\left(\xi_{1}-a_{i}+\gamma\right)}{w^{2}\left(\xi_{1}\right)}\right|}{C} \rightarrow 0, \quad C \rightarrow \infty,
\end{aligned}
$$

whence

$$
\varepsilon_{1, n} \stackrel{\mathrm{P}}{\rightarrow} 0, \quad n \rightarrow \infty .
$$

By the mean value theorem,

$$
\begin{aligned}
\varepsilon_{2, n} & =p \frac{1}{n} \sum_{j=1}^{n} \frac{u_{k}^{\prime}\left(\xi_{j}-\hat{a}_{1, n}\right)-u_{k}^{\prime}\left(\xi_{j}-a_{1}\right)}{w\left(\xi_{j}\right)} \\
& =p\left(a_{1}-\hat{a}_{1, n}\right) \frac{1}{n} \sum_{j=1}^{n} \frac{u_{k}^{\prime \prime}\left(\xi_{j}-a_{1}+\theta\left(a_{1}-\hat{a}_{1, n}\right)\right)}{w\left(\xi_{j}\right)} .
\end{aligned}
$$

Similarly to the case of $\varepsilon_{1, n}$, restriction 2 of Theorem 6.1 implies that

$$
\varepsilon_{2, n} \stackrel{\mathrm{P}}{\rightarrow} 0, \quad n \rightarrow \infty .
$$

The strong law of large numbers and restriction 1 of Theorem 6.1 imply that

$$
\varepsilon_{3, n} \stackrel{\mathrm{P}}{\rightarrow} 0, \quad n \rightarrow \infty
$$

whence $\hat{b}_{k, n}^{+} \stackrel{\mathrm{P}}{\rightarrow} b_{k}^{+}, n \rightarrow \infty$.

Analogously $\hat{b}_{k, n}^{-} \stackrel{\mathrm{P}}{\rightarrow} b_{k}^{-}, n \rightarrow \infty$.

Next,

$$
\begin{aligned}
\hat{d}_{k, n}-d_{k}= & {\left[\frac{1}{n} \sum_{j=1}^{n} \frac{u_{k}\left(\xi_{j}-\hat{a}_{2, n}\right)}{w\left(\xi_{j}\right)}-\mathrm{E} \frac{u_{k}\left(\xi_{1}-a_{2}\right)}{w\left(\xi_{1}\right)}\right] } \\
& -\left[\frac{1}{n} \sum_{j=1}^{n} \frac{u_{k}\left(\xi_{j}-\hat{a}_{1, n}\right)}{w\left(\xi_{j}\right)}-\mathrm{E} \frac{u_{k}\left(\xi_{1}-a_{1}\right)}{w\left(\xi_{1}\right)}\right]=\varepsilon_{4, n}-\varepsilon_{5, n},
\end{aligned}
$$




$$
\begin{aligned}
\varepsilon_{4, n} & =\left[\frac{1}{n} \sum_{j=1}^{n} \frac{u_{k}\left(\xi_{j}-\hat{a}_{2, n}\right)}{w\left(\xi_{j}\right)}-\mathrm{E} \frac{u_{k}\left(\xi_{1}-a_{2}\right)}{w\left(\xi_{1}\right)}\right] \\
& =\left[\frac{1}{n} \sum_{j=1}^{n} \frac{u_{k}\left(\xi_{j}-\hat{a}_{2, n}\right)}{w\left(\xi_{j}\right)}-\frac{1}{n} \sum_{j=1}^{n} \frac{u_{k}\left(\xi_{j}-a_{2}\right)}{w\left(\xi_{j}\right)}\right] \\
& +\left[\frac{1}{n} \sum_{j=1}^{n} \frac{u_{k}\left(\xi_{j}-a_{2}\right)}{w\left(\xi_{j}\right)}-\mathrm{E} \frac{u_{k}\left(\xi_{1}-a_{2}\right)}{w\left(\xi_{j}\right)}\right] \stackrel{\mathrm{P}}{\rightarrow} 0, \quad n \rightarrow \infty,
\end{aligned}
$$

since the first term tends to zero in probability by restriction 1 of the theorem and the second term tends to zero in probability by the strong law of large numbers and restriction 3 of the theorem.

Analogously, $\varepsilon_{5, n} \stackrel{\mathrm{P}}{\rightarrow} 0$ as $n \rightarrow \infty$ and thus $\hat{d}_{k, n} \stackrel{\mathrm{P}}{\rightarrow} d_{k}$ as $n \rightarrow \infty$.

Now we prove that the estimators $\hat{c}_{k l, n}$ are consistent. We have

$$
\hat{c}_{k l, n}-c_{k l}=\delta_{1}+\delta_{2}+\delta_{3}+\delta_{4},
$$

where

$$
\begin{gathered}
\delta_{1}=\frac{\hat{p}_{n}^{2}}{n} \sum_{j=1}^{n} u_{k}\left(\xi_{j}-\hat{a}_{1, n}\right) u_{l}\left(\xi_{j}-\hat{a}_{1, n}\right)-p^{2} \mathrm{E} u_{k}\left(\xi_{j}-a_{1}\right) u_{l}\left(\xi_{j}-a_{1}\right), \\
\delta_{2}=\frac{\hat{p}_{n}\left(1-\hat{p}_{n}\right)}{n} \sum_{j=1}^{n} u_{k}\left(\xi_{j}-\hat{a}_{1, n}\right) u_{l}\left(\xi_{j}-\hat{a}_{2, n}\right)-p(1-p) \mathrm{E} u_{k}\left(\xi_{j}-a_{1}\right) u_{l}\left(\xi_{j}-a_{2}\right), \\
\delta_{3}=\frac{\hat{p}_{n}\left(1-\hat{p}_{n}\right)}{n} \sum_{j=1}^{n} u_{k}\left(\xi_{j}-\hat{a}_{2, n}\right) u_{l}\left(\xi_{j}-\hat{a}_{1, n}\right)-p(1-p) \mathrm{E} u_{k}\left(\xi_{j}-a_{2}\right) u_{l}\left(\xi_{j}-a_{1}\right), \\
\delta_{4}=\frac{\left(1-\hat{p}_{n}\right)^{2}}{n} \sum_{j=1}^{n} u_{k}\left(\xi_{j}-\hat{a}_{2, n}\right) u_{l}\left(\xi_{j}-\hat{a}_{2, n}\right)-(1-p)^{2} \mathrm{E} u_{k}\left(\xi_{j}-a_{2}\right) u_{l}\left(\xi_{j}-a_{2}\right) .
\end{gathered}
$$

We are going to show that $\delta_{1} \rightarrow 0$. The proof of $\delta_{4} \rightarrow 0$ is similar. We have

$$
\delta_{1}=\varepsilon_{5, n}+\varepsilon_{6, n}+\varepsilon_{7, n},
$$

where

$$
\begin{gathered}
\varepsilon_{5, n}=\frac{\hat{p}_{n}^{2}}{n} \sum_{j=1}^{n} \frac{u_{k}\left(\xi_{j}-\hat{a}_{1, n}\right) u_{l}\left(\xi_{j}-\hat{a}_{1, n}\right)-u_{k}\left(\xi_{j}-a_{1}\right) u_{l}\left(\xi_{j}-a_{1}\right)}{w^{2}\left(\xi_{j}\right)}, \\
\varepsilon_{6, n}=\left(\hat{p}_{n}^{2}-p^{2}\right) \frac{1}{n} \sum_{j=1}^{n} \frac{u_{k}\left(\xi_{j}-a_{1}\right) u_{l}\left(\xi_{j}-a_{1}\right)}{w^{2}\left(\xi_{j}\right)}, \\
\varepsilon_{7, n}=\frac{p^{2}}{n} \sum_{j=1}^{n} \frac{u_{k}\left(\xi_{j}-a_{1}\right) u_{l}\left(\xi_{j}-a_{1}\right)}{w^{2}\left(\xi_{j}\right)}-p^{2} \mathrm{E} \frac{u_{k}\left(\xi_{1}-a_{1}\right) u_{l}\left(\xi_{1}-a_{1}\right)}{w^{2}\left(\xi_{1}\right)} .
\end{gathered}
$$

By the law of large numbers,

$$
\varepsilon_{7, n} \stackrel{\mathrm{P}}{\rightarrow} 0, \quad n \rightarrow \infty .
$$

Since $\hat{p}_{n}^{2}-p^{2} \stackrel{\mathrm{P}}{\rightarrow} 0$ as $n \rightarrow \infty$ and since the sequence

$$
\frac{1}{n} \sum_{j=1}^{n} \frac{u_{k}\left(\xi_{j}-a_{1}\right) u_{l}\left(\xi_{j}-a_{1}\right)}{w^{2}\left(\xi_{j}\right)}
$$


is stochastically bounded by restriction 3 , we get

$$
\varepsilon_{6, n} \stackrel{P}{\rightarrow} 0, \quad n \rightarrow \infty .
$$

Let $Z=u_{k}^{\prime} u_{l}+u_{k} u_{l}^{\prime}$. Consider

$$
\varepsilon_{5, n}=\left(a_{1}-\hat{a}_{1, n}\right) \frac{\hat{p}_{n}^{2}}{n} \sum_{j=1}^{n} \frac{Z\left(\xi_{j}-a_{1}+\theta\left(a_{1}-\hat{a}_{1, n}\right)\right)}{w^{2}\left(\xi_{j}\right)},
$$

where $\theta \in[0,1]$. Since

$$
\frac{1}{n} \sum_{j=1}^{n} \frac{\left(u_{k}^{\prime} u_{l}+u_{k} u_{l}^{\prime}\right)}{w^{2}\left(\xi_{j}\right)} \leq \sqrt{\frac{1}{n} \sum_{j=1}^{n}\left(\frac{u_{k}^{\prime} u_{l}}{w^{2}\left(\xi_{j}\right)}\right)^{2}}+\sqrt{\frac{1}{n} \sum_{j=1}^{n}\left(\frac{u_{k} u_{l}^{\prime}}{w^{2}\left(\xi_{j}\right)}\right)^{2}},
$$

this sequence is stochastically bounded by restrictions 1 and 3 . Hence $\hat{p}_{n}^{2}$ also is stochastically bounded and $\hat{a}_{1, n}-a_{1} \stackrel{\mathrm{P}}{\rightarrow} 0, n \rightarrow \infty$. Therefore

$$
\varepsilon_{5, n} \stackrel{\mathrm{P}}{\rightarrow} 0, \quad n \rightarrow \infty .
$$

Relations (11)-(13) imply that

$$
\delta_{1} \stackrel{\mathrm{P}}{\rightarrow} 0, \quad n \rightarrow \infty .
$$

The term $\delta_{4}$ is similar to $\delta_{1}$ and thus the proof of $\delta_{4} \stackrel{\mathrm{P}}{\rightarrow} 0$ as $n \rightarrow \infty$ is the same.

Let $\delta_{2}=\varepsilon_{8, n}+\varepsilon_{9, n}+\varepsilon_{10, n}$, where

$$
\begin{gathered}
\varepsilon_{9, n}=\frac{\hat{p}_{n}\left(1-\hat{p}_{n}\right)}{n} \sum_{j=1}^{n}\left[\frac{u_{k}\left(\xi_{j}-\hat{a}_{1, n}\right) u_{l}\left(\xi_{j}-\hat{a}_{2, n}\right)-u_{k}\left(\xi_{j}-a_{1}\right) u_{l}\left(\xi_{j}-a_{2}\right)}{w^{2}\left(\xi_{j}\right)},\right. \\
\varepsilon_{10, n}=\frac{\left(\hat{p}_{n}-p\right)\left(1-\hat{p}_{n}-p\right)}{n} \sum_{j=1}^{n} \frac{u_{k}\left(\xi_{j}-a_{1}\right) u_{l}\left(\xi_{j}-a_{2}\right)}{w^{2}\left(\xi_{j}\right)}, \\
\varepsilon_{11, n}=\frac{p(1-p)}{n}\left[\sum_{j=1}^{n} \frac{u_{k}\left(\xi_{j}-a_{1}\right) u_{l}\left(\xi_{j}-a_{2}\right)}{w^{2}\left(\xi_{j}\right)}-\mathrm{E} \frac{u_{k}\left(\xi_{1}-a_{1}\right) u_{l}\left(\xi_{1}-a_{2}\right)}{w^{2}\left(\xi_{1}\right)}\right] .
\end{gathered}
$$

By the law of large numbers,

$$
\varepsilon_{11, n} \stackrel{\mathrm{P}}{\rightarrow} 0, \quad n \rightarrow \infty .
$$

The term $\varepsilon_{10, n}$ is a product of a stochastically bounded sequence and an infinitesimal in probability sequence. Hence

$$
\varepsilon_{10, n} \stackrel{\mathrm{P}}{\rightarrow} 0, \quad n \rightarrow \infty .
$$

Similarly to the case of $\varepsilon_{5, n}$,

$$
\begin{aligned}
& \varepsilon_{9, n}= \frac{\hat{p}_{n}\left(1-\hat{p}_{n}\right)}{n}\left(a_{2}-\hat{a}_{2, n}\right)\left(a_{1}-\hat{a}_{1, n}\right) \\
& \times \sum_{j=1}^{n}\left[\frac{u_{k}^{\prime}\left(\xi_{j}-a_{1}+\theta_{1}\left(a_{1}-\hat{a}_{1, n}\right)\right) u_{l}\left(\xi_{j}-a_{2}+\theta_{2}\left(a_{2}-\hat{a}_{2, n}\right)\right)}{w^{2}\left(\xi_{j}\right)}\right. \\
&\left.\quad+\frac{u_{k}\left(\xi_{j}-a_{1}+\theta_{1}\left(a_{1}-\hat{a}_{1, n}\right)\right) u_{l}^{\prime}\left(\xi_{j}-a_{2}+\theta_{2}\left(a_{2}-\hat{a}_{2, n}\right)\right)}{w^{2}\left(\xi_{j}\right)}\right],
\end{aligned}
$$

where $\theta_{1}, \theta_{2} \in[0,1]$ are random variables.

Restrictions 1 and 3 imply that

$$
\varepsilon_{9, n} \stackrel{\mathrm{P}}{\rightarrow} 0, \quad n \rightarrow \infty,
$$


whence

$$
\delta_{2} \stackrel{\mathrm{P}}{\rightarrow} 0, \quad n \rightarrow \infty .
$$

The proof of $\delta_{3} \stackrel{\mathrm{P}}{\rightarrow} 0$ as $n \rightarrow \infty$ is the same.

The proof of Theorem 6.1 is complete.

Corollary 6.1. If the assumptions of Theorem 6.1 hold and if $\operatorname{det} C \neq 0$, then

$$
\hat{\beta}_{i} \stackrel{\mathrm{P}}{\rightarrow} \beta_{i}^{*}, \quad i=1, \ldots, 3,
$$

where the $\hat{\beta}_{i}$ are columns of the matrix $\left(\hat{B}^{T} \hat{C}^{-1} \hat{B}\right)^{-1} \hat{C}^{-1} \hat{B}$.

Proof. The theorem implies that $\hat{B} \stackrel{\mathrm{P}}{\rightarrow} B$ and $\hat{C} \stackrel{\mathrm{P}}{\rightarrow} C$ in the sense that every element of a prelimit matrix tends to the corresponding element of the limit matrix. Thus

$$
\hat{\beta}_{n}=\left(\hat{B}^{T} \hat{C}^{-1} \hat{B}\right)^{-1} \hat{C}^{-1} \hat{B} \stackrel{\mathrm{P}}{\rightarrow}\left(B^{T} C^{-1} B\right)^{-1} C^{-1} B=\beta^{*} .
$$

\section{Adaptive estimators of EuClidean parameters}

Analogously to [5], we construct adaptive estimators of parameters of a mixture that have a minimal asymptotic covariance matrix.

According to Theorem 6.1, one can construct consistent estimators $\left\{\hat{\beta}_{i m}\right\}$ for the optimal coefficients

$$
\left\{\beta_{i m}^{*}\right\}, \quad i=1, \ldots, 3, m=1, \ldots, M .
$$

Substituting estimators in (77) we obtain estimators for the optimal estimating functions

$$
\mathbf{g}_{\hat{\beta}_{i}}(x)=\sum_{m=1}^{M} \hat{\beta}_{i m} u_{m}(x) .
$$

Substituting (14) in (8) we get the adaptive system of equations

$$
\pi \hat{\mathbf{g}}_{\hat{\beta}_{i}}\left(\alpha_{1}\right)+(1-\pi) \hat{\mathbf{g}}_{\hat{\beta}_{i}}\left(\alpha_{2}\right)=0, \quad i=1,2,3 .
$$

Denoting the left-hand side of system (15) by

$$
\hat{\mathbf{h}}_{\hat{\beta}}(t)=\left(\begin{array}{l}
\pi \hat{\mathbf{g}}_{\hat{\beta}_{1}}\left(\alpha_{1}\right)+(1-\pi) \hat{\mathbf{g}}_{\hat{\beta}_{1}}\left(\alpha_{2}\right) \\
\pi \hat{\mathbf{g}}_{\hat{\beta}_{2}}\left(\alpha_{1}\right)+(1-\pi) \hat{\mathbf{g}}_{\hat{\beta}_{2}}\left(\alpha_{2}\right) \\
\pi \hat{\mathbf{g}}_{\hat{\beta}_{3}}\left(\alpha_{1}\right)+(1-\pi) \hat{\mathbf{g}}_{\hat{\beta}_{3}}\left(\alpha_{2}\right)
\end{array}\right), \quad t=\left(\begin{array}{c}
\pi \\
\alpha_{1} \\
\alpha_{2}
\end{array}\right),
$$

we have an equivalent vector estimating equation

$$
\hat{\mathbf{h}}(t)=0 .
$$

It is not easy to solve this equation and thus we construct some approximations to its solution. Let

$$
\tilde{\theta}=\left(\begin{array}{c}
\tilde{p}_{n} \\
\tilde{a}_{1} \\
\tilde{a}_{2}
\end{array}\right)
$$

be a pilot estimator of the parameter $\theta=\left(p, a_{1}, a_{2}\right)^{T}$ that is $\sqrt{n}$-consistent. One of the possible choices here is the method of moments estimator. Consider the Taylor expansion of the left-hand side of (16) in a neighborhood of the point $\tilde{\theta}_{n}$ :

$$
\hat{\mathbf{h}}_{\hat{\beta}}(t) \approx \hat{\mathbf{h}}_{\hat{\beta}}\left(\tilde{\theta}_{n}\right)+\hat{\mathbf{h}}_{\hat{\beta}}^{\prime}\left(\tilde{\theta}_{n}\right)\left(t-\tilde{\theta}_{n}\right)=0,
$$

where $\hat{\mathbf{h}}_{\hat{\beta}}^{\prime}(t)$ is the Jacobi matrix,

$$
\hat{\mathbf{h}}_{\hat{\beta}}^{\prime}(t)=\frac{D\left(h_{1}, 2, h_{3}\right)}{D\left(\pi, \alpha_{1}, \alpha_{2}\right)} .
$$


Solving this equation with respect to $t$ and taking into account that $\hat{\mathbf{h}}_{\hat{\beta}}^{\prime}(\theta) \approx E$ by the normalization condition in Theorem 3.2 of [5], we obtain an approximate adaptive estimator of the unknown parameter

$$
\breve{\theta}_{n}=\tilde{\theta}_{n}-\hat{\mathbf{h}}_{\hat{\beta}}\left(\tilde{\theta}_{n}\right)
$$

Theorem 7.1. Assume that

1. the derivatives $f^{\prime}(x)$ and $u_{m}^{\prime}(x), m=1, \ldots, M$, are continuous;

2. $\hat{\theta}_{n}=\left(\hat{p}_{n}, \hat{a}_{1, n}, \hat{a}_{2, n}\right)^{T}$ is a consistent estimator;

3. $\mathrm{E} \frac{u_{m}^{2}\left(\xi_{1}-a_{k}\right)}{w^{2}\left(\xi_{1}\right)}<\infty, m=1, \ldots, M$ and $k=1,2$;

4. all entries of the matrix $V$ are finite; $\operatorname{det} V \neq 0$;

5 .

$$
\begin{gathered}
\exists \varepsilon>0, \delta>0: \quad \mathrm{E} \sup _{\alpha:\left|\alpha-a_{k}\right|<\varepsilon}\left|\frac{u_{m}\left(\xi_{1}-\alpha\right)}{w\left(\xi_{1}\right)}\right|^{1+\delta}<\infty, \\
\mathrm{E} \sup _{\alpha:\left|\alpha-a_{k}\right|<\varepsilon}\left|\frac{u_{m}^{\prime}\left(\xi_{1}-\alpha\right)}{w\left(\xi_{1}\right)}\right|^{1+\delta}<\infty, \quad m=1, \ldots, M, \quad k=1,2 ;
\end{gathered}
$$

6. $u_{m}(x+\delta) f(x) \rightarrow 0$ as $x \rightarrow \pm \infty$ for all $\delta \in \mathbf{R}$;

7. $w(x)>0, x \in \operatorname{supp}(F)$.

Then $\sqrt{n}\left(\breve{\theta}_{n}-\theta\right) \stackrel{d}{\rightarrow} N\left(0, \Sigma^{*}\right), n \rightarrow \infty$.

The proof of this theorem is similar to that of Theorem 5.1 in $[5]$.

\section{ACKNOWLEDGEMENT}

The author is grateful to the scientific advisor R. E. Mauboroda for the setting of the problem and discussion of results.

\section{BIBLIOGRAPHY}

1. D. M. Titterington, A. F. M. Smith, and U. E. Makov, Statistical Analysis of Finite Mixture Distribution, Wiley, New York, 1985. MR838090 (87j:62033)

2. G. J. McLachlan and D. Peel, Finite Mixture Models, Wiley-Interscience, 2000. MR1789474 (2002b:62025)

3. R. E. Maiboroda, Estimation of mean positions and concentrations from observations of a twocomponent mixture of symmetric distributions, Teor. Imovir. Matem. Statist. 78 (2008), 133141; English transl. in Theor. Probability and Math. Statist. 78 (2009), 147-156. MR2446855 (2010b:62134)

4. R. E. Maıboroda and O. Sugakova, Estimate for Euclidean parameters of a mixture of two symmetric distributions, Ukr. Matem. Zh. 62 (2010), no. 7, 945-953; English transl. in Ukrain. Math. J. 62 (2010), no. 7, 1098-1108. MR.2888662

5. O. Sugakova, Adaptive estimators for parameters of a mixture of two symmetric distributions, Teor. Imovir. Matem. Statist. 82 (2010), 146-155; English transl. in Theor. Probability and Math. Statist. 82 (2011), 149-159. MR2790490 (2011m:62130)

6. D. R. Hunter, S. Wang, and T. R. Hettmansperger, Inference for mixtures of symmetric distributions, Ann. Statist. 35 (2007), 224-251. MR2332275 (2008g:62079)

7. J. Shao, Mathematical Statistics, Springer-Verlag, New York, 1998. MR2002723 (2004g:62002)

8. S. L. Lohr, Sampling: Design and Analysis, Duxbury Press, 1999. MR3057878

9. O. I. Vasylyk and T. O. Yakovenko, Lectures in the Theory of Sampling Methods, Kyiv University Press, Kyiv, 2010. (Ukrainian)

10. M. V. Kartashov, Probability, Processes, Statistics, Kyiv University Press, Kyiv, 2007. (Ukrainian) 
Department of Probability Theory, Statistics, and Actuarial Mathematics, Faculty for Mechanics and Mathematics, National Taras Shevchenko University, Volodymyrs'ka Street, 64, KYIV 01601, UKRAINE

Received 31/JULY/2013

Translated by S. KVASKO 